\title{
Palliative wound treatment promotes healing in hospice
}

\author{
Aletha W. Tippett * \\ Advanced Wound Team, Cincinnati, Ohio, United State
}

Received: September 21, 2014

Accepted: December 28, 2014 Online Published: January 8, 2015

DOI: $10.5430 /$ cns.v3n2p53

URL: http://dx.doi.org/10.5430/cns.v3n2p53

\begin{abstract}
Introduction: Wounds are a major problem at the end of life, affecting nearly one third of hospice patients. The main problems with these wounds are pain, infection and odor, making wound care often difficult.

Method: A method of wound treatment invented in 2001 for palliative wound care was designed to meet the goals of pain relief, odor control, and prevention of infection. This method, which involved application of viscous lidocaine and topical polymyxin/bacitracin to gauze, was used on hundreds of hospice patients. A retrospective review of results was undertaken.

Results: A descriptive retrospective observational review is presented here. This is a retrospective review of 323 wounds in hospice patients treated over a period of 30 months using the palliative wound treatment method. In this cohort over $40 \%$ of pressure ulcers healed to closure or were healing, $30 \%$ of ischemic ulcers were healed to closure or were healing and over $40 \%$ of neuropathic ulcers were healing, totaling nearly $40 \%$ of all wounds healing or healed to closure.
\end{abstract}

Conclusion: While meeting palliative goals of reducing pain, preventing infection and controlling odor, use of this method also provided unexpected healing. Further investigation on use of this method is warranted.

Key Words: Pressure ulcer, Palliative wound care, Wound pain, Wound odor, Wound infection

\section{Introduction}

Published data report $35 \%$ of hospice patients have wounds. ${ }^{[1,2]}$ Of those wounds, $50 \%$ are pressure ulcers and $20 \%$ are ischemic. ${ }^{[1,2]}$ The remaining $30 \%$ is a mix of various wounds, including surgical, stasis, skin tears, and tumors. $^{[1]}$ With nearly 2 million patients in hospice in the U.S. currently, ${ }^{[3]}$ this correlates to approximately 700,000 wounds needing care.

Most chronic wounds are pressure ulcers, also known as bedsores or decubiti. Pressure ulcers are a tragic problem for millions of Americans. Five to seven million Americans develop a chronic wound each year, with the incidence increasing $10 \%$ per year, ${ }^{[4]}$ of which 2.5 million of these chronic wounds are pressure ulcers, remarkably close to the $50 \%$ noted in hospice ${ }^{[1,5]}$ Pressure ulcers are a significant problem for frail and elderly people, ${ }^{[6]}$ but also for people who are paralyzed either from spinal cord or brain injury ${ }^{[7]}$ or neurodegenerative diseases (such as multiple sclerosis or Huntington's). ${ }^{[8]}$ Pressure ulcers typically occur over a bony prominence that has unrelieved pressure from normal gravitational body force, ${ }^{[9]}$ such as the sacrum in bed-bound patients, ischial tuberosities from sitting, and on heels from lying in bed. In the United States alone the pressure ulcer burden is staggering costing in excess of $\$ 9.1$ billion for treatment annually. ${ }^{[5]}$ These pressure ulcers are fullthickness, often very deep, painful, and prone to infection. Most commonly these wounds are located on the sacrum or

*Correspondence: Dr. Aletha W. Tippett, MD; Email: tippettaw @fuse.net; Address: 10274 Alliance Road, Cincinnati, OH 45242, United State. 
in the pelvic region. ${ }^{[1]}$ The location of the wound in this region can make treatment very difficult. Contamination with urine and feces is a frequent problem. Fastening a dressing to the wound may be quite difficult due to size requirements, body shape, moisture, fragile/thin skin that tears easily, or presence of body hair.

An effective dressing to adequately treat these wounds is highly desirable and most providers would agree that there is no single dressing that works that well. ${ }^{[10,11]}$ Wounds and wound care are fraught with pain, yet few dressings provide much needed pain relief. For patients with chronic wounds, pain has been described as the worst part of living with a chronic wound. ${ }^{[12]}$ Pain is a complex biopsychosocial phenomenon that requires multiple pharmacological and nonpharmacological management approaches. ${ }^{[12]}$

All open wounds are contaminated with bacteria which can lead to infection, and wound infection is a scourge to patients and caregivers, with some estimates as high as $30 \%$ of wounds affected. ${ }^{[13]}$ A wound dressing with antimicrobial activity against common wound pathogens would be very advantageous.

For hospice patients at the end of life, goals of care are pain relief, infection prevention, and providing comfort and dignity. Healing of wounds or wound closure are not primary goals as the patient's life span is very short, on average only about 67 days. ${ }^{[3]}$ In 2001, a method was developed by a hospice physician to meet the palliative objectives of pain reduction, odor control, and infection prevention for wounds in hospice patients. Since 2001 this method has been in use on hundreds, if not thousands, of patients in hospice and later for patients not in hospice.

\section{Development of wound treatment}

The current method was developed in 2001 at the bedside primarily to provide pain relief in dying patients with painful pressure ulcers. At that time there were no guidelines for palliative wound care, and wound healing was seen as an unrealistic goal of treatment in this patient population. ${ }^{[14]}$ This technique was not developed from clinical trials or laboratory review, but from a clinical and pharmacological knowledge base of using lidocaine for pain relief, with pain relief desired for palliative wound care. The method involved application of viscous lidocaine and a topical antibiotic to gauze.

Prior to use, an extensive literature search was conducted to investigate the use of lidocaine in wounds. Animal studies $^{[15-19]}$ showed mixed results for adequate healing in wounds treated with lidocaine. Human studies using lidocaine for burns showed that it was safe to use topically on a wound, being below toxicity level. ${ }^{[20,21]}$ Nothing in the literature was found to either support or contradict use of lidocaine in the wound bed for pain relief, yet it is known that topical lidocaine can decrease pain. ${ }^{[22]}$ Great care was taken to ensure that the amounts of lidocaine used were below toxicity level. Brofeldt et al. studied application of $5 \%$ lidocaine to burns, on up to $28 \%$ of total body surface area. Serum levels never reached toxicity level. ${ }^{[20]}$ This method discussed here has less than $2 \%$ lidocaine in application, so is safe for very large wound areas, greater than $28 \%$ body surface area (BSA). A viscous lidocaine was selected because of its known human safety, its low percentage of lidocaine, and because it contains carboxymethylcellulose which makes a hydrogel that adheres well in an open wound. Hydrogels have an observed benefit in treating various types of wounds, believed to be due to the promotion of the body's intrinsic autolysis process. ${ }^{[23,24]}$ When viscous lidocaine was used the mixture contained approximately $1 \%$ lidocaine, an amount that has shown safe reduction of pain when applied on a wound. ${ }^{[22]}$

Because of the theoretical risk of increased infection due to an altered inflammatory response, ${ }^{[25]}$ antibiotics were added with the lidocaine. A simple mixture of polymyxin and bacitracin was used based on reports suggesting this may improve wound healing. ${ }^{[26]}$ In vitro efficacy testing later performed on this mixture showed inhibition of four of the major wound pathogens: Escherichia coli, Staphylococcus aureus, Candida albicans, and Pseudomonas aeruginosa. ${ }^{\text {[27] }}$ Added antibiotics give good inhibitory effects against major wound pathogens, but the presence of lidocaine can also contribute to antibacterial effects and bacterial minimization. ${ }^{[28,29]}$

Malodor is a known problem with wounds but the general consensus at the time of this invention was good cleansing and treating infection. Using an agent such as topical metronidazole for odor was first reported in $1993,{ }^{[30]}$ but was not yet standard of care at the time of development of this method. For the population in this treatment cohort, relieving the patient of the burden of foul odor was paramount. With that rationale, oil of wintergreen was added, using several drops for each wound treated. Oil of wintergreen has a strong and cooling aroma, and it contains 85\%-99\% methyl salicylate which is aspirin-like and can contribute to pain relief as promoted in commercial literature. ${ }^{[31]}$ The main ingredient in oil of wintergreen, methyl salicylate, has much scientific information available from the National Center for Biotechnology Information, published in a compound summary. ${ }^{[32]}$ Since 2001 there has been more study on options for controlling odor with different agents. ${ }^{[33,34]}$

An important characteristic is that wound care was simplified as the procedure was useful for any type of wound. It was not necessary to characterize the wound. Depending on the wound dressed and the top dressing used, it required changing every 1-7 days.

\section{Method}

This is a retrospective observational chart review of 192 hospice patients with 323 wounds treated over 30 months. This 
study is from previously published data and results, ${ }^{[1]}$ but this report details specific treatment not covered in the previous report. Types of wounds included pressure, neuropathic, ischemic and other. Wound treatment selection was determined by hospice physician recommendation and patient/caregiver consent. Consent was obtained as part of standard practice for any treatment offered. With patient or caregiver permission, wounds were treated with the mixture of lidocaine and topical antibiotic, moistened as needed with normal saline, applied on a gauze to fit the wound. Wound dressings were changed daily, sometimes less frequently if the wound was dry, or more frequently if there was heavy drainage. Not all patients or caregivers gave permission for use of this treatment; some were afraid of using lidocaine in the wound; some did not perceive it as aggressive treatment; some just thought it seemed too unusual to work, therefore there were $92(28 \%)$ wounds treated with another modality based on whatever their physician had ordered. The other wound treatments included many other types of dressing per the choice of the treating physician, such as wet to dry, hydrocolloids, alginates, or vacuum assisted therapy. 231 (72\%) wounds were treated with the new method, which hence will be referred to as the novel dressing. Ninetytwo (92) wounds were treated with other methods. Wound healing was judged by the traditional method of measuring length and width, judging the amount of drainage, and assessing the wound tissue for granulation, slough, or necrosis. A wound was judged to be healing if it showed contracture with granulation and decreased size. Wounds were assessed weekly by a nurse, and every 1-3 weeks by the hospice physician. Since the palliative goals of care were to relieve pain, prevent infection, and control odor, these were evaluated with each assessment. Since the majority of patients were non-verbal no analog scales were used; evaluations depended on nursing assessments. Pain assessments were made by observing patient facial expressions and body movements and any moaning or crying.

This method provided a primary wound dressing, so needed a secondary dressing. In keeping with the desire for simplicity and maintaining comfort, the top dressing was provided by applying a thick layer of zinc oxide ointment around the wound, then topping the wound with a sheet of plastic wrap, pressed into the zinc oxide ointment.

\section{Results}

The median age of patients treated was 82. Most patients lived less than 30 days, the median was 31 days. Healing to closure was not an end-point in treatment of this cohort as most of the patients would not live long enough to go to closure. By nursing reports, odor and pain were reduced. Pain relief was immediate in most cases, and persisted with use of the novel dressing. In the cases treated, no infections occurred with use of the dressing. Odor was also well controlled. Besides reducing pain, preventing infection and controlling odor, healing results were surprising. Over $40 \%$ of pressure ulcers healed to closure or were healing, $30 \%$ of ischemic ulcers were healed to closure or were healing and over $40 \%$ of neuropathic ulcers were healing. There were no allergic reactions seen. Allergic reaction to lidocaine has been found to be low $(<0.7 \%){ }^{[22]}$ The top dressing used was very successful. It kept the wound dressing moist and warm, both adjuvants to healing, ${ }^{[35]}$ and prevented trauma to periwound skin with dressing changes.

See Figure 1 for results in the treatment of 231 wounds in hospice patients. This is a bar graph showing percent wounds healing or healed to closure and the percent stable, meaning no change in size. This graph shows the three most prevalent wound types treated, pressure, arterial (ischemic), and neuropathic (diabetic).

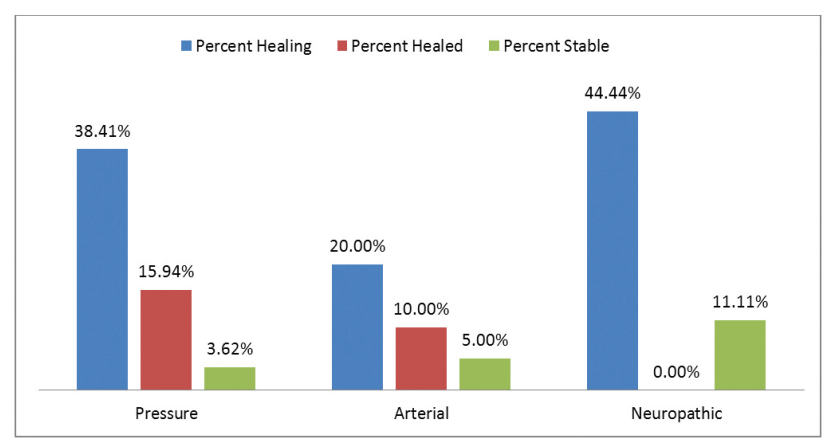

Figure 1: Palliative wound treatment healing results by wound type

Included in this study were 24 diabetic foot wounds treated over the 30 month period, 11/24 (46\%) healed to closure or were healing, no new wound infections presented, and there were no amputations.

A comparison of 231 wounds treated with the novel dressing versus the 92 wounds receiving other treatments is shown in Figure 2. Overall, $40 \%$ of wounds treated with the novel dressing healed to closure or were healing vs. $10 \%$ of wounds treated with other modalities.

Since the novel dressing uses gauze it is interesting to look at a published study of VAC vs. moist gauze healing, ${ }^{[36]}$ seen in Figure 3. Wound volume reduction was assessed at 3 and 6 weeks in 18 patients with chronic non healing sacral/pelvic ulcers in each arm of the study. While no direct comparison can be made, twelve hospice patients from this study with sacral/pelvic ulcers that were examined at 3 and 6 weeks were compared to the published study. Because of the difference in sample sizes no direct comparison can be made, but results are intriguing, nevertheless, especially since the VAC and gauze arms excluded patients with malnutrition, renal failure, infection, cancer or other chronic disease requiring treatment and the novel dressing arm had no exclusions. Patients in the novel dressing arm were also much older, on average. 


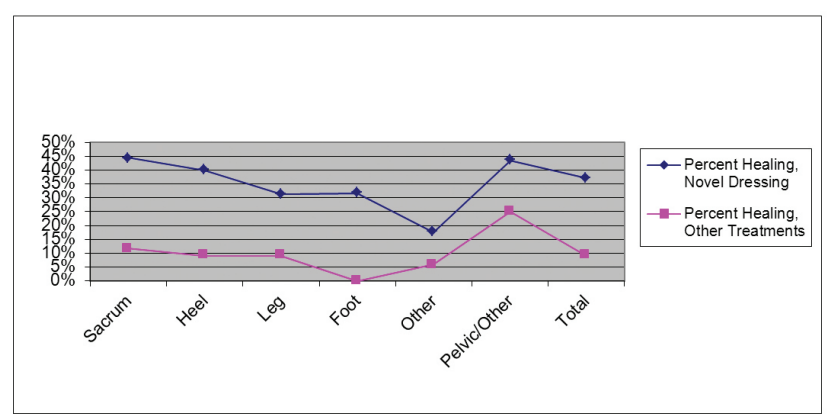

Figure 2: Comparison of healing rates of novel dressing $v s$. other treatments

\section{Discussion}

The wound treatment under discussion is a novel dressing using common, ordinary materials in an uncommon way. The ingredients used to make this dressing were gauze, viscous lidocaine, polymyxin/bacitracin antibiotic and oil of wintergreen. Gauze was the substrate, often discounted, but yet very versatile and useful. Gauze can be shaped to any size or form, can be used to moisten or dry a wound, and can easily be combined with other products. Viscous lidocaine not only provided lidocaine for pain relief, but the viscous part is a hydrogel which brings all the advantages of a hydrogel such as maintaining moisture and promoting autolytic debridement. It also controls the $\mathrm{pH}$, making a slightly acidic wound dressing. ${ }^{[37]}$ The antibiotic used has broad antibacterial activity and hastened wound healing in one study. ${ }^{[26]}$ Also, in vitro studies with the novel dressing show inhibition of some common wound pathogens, including E coli, $\mathrm{S}$ aureus, $\mathrm{P}$ aeruginosa and $\mathrm{C}$. albicans. ${ }^{[27]}$ The oil of wintergreen is a natural oil added in small amounts for odor control. In both of the reviews reported using the novel dressing there were no new infections, odor was controlled, and pain was relieved or reduced.

While the original goals of treating pain and preventing infection were met, one surprising outcome was improved healing of wounds. Results have been beyond initial expectations for this method. Initially there was no goal of healing in dying patients, just pain relief, prevention of infection and control of odor. Surprisingly, wounds however improved and even healed in this very fragile group of patients. All the wounds had prior treatment, some with surgery, and all with any variety of dressings, most with multiple different dressings, such as wet to dry gauze, hydrocolloids, alginates, silver dressings, or vacuum-assisted wound dressing. In all cases, this was the first treatment that had worked to help heal the wound. And in all cases, healing was not expected because these were dying patients, some with "terminal wounds". ${ }^{[38]}$ Mostly the results seen with this treatment method were surprising to observers, with over $40 \%$ of the wounds healed or healing. Success with hospice patients caused use of this method to spread to non-hospice patients as well. Results of healing in the hundreds of wounds treated with this method are here being compared to reported results in other studies. When reviewing these results one finds remarkable healing compared to other art in the field.

This study is observational, not experimental. Because assignment of treatment was not random, but elected by the patient or caregiver, this study is actually "quasiexperimental". All individuals were simply observed to have the intervention or not, and outcomes subsequently assessed. Data collected was primary data collected by the investigator. ${ }^{[39]}$ Even though this was observational research, one still must consider issues of bias, precision, validity, confounding and chance. In cohort design such as in this study, selection bias by the investigator must be considered, but in this case the investigator did not select participants, they selected themselves by consenting or no. Confounding can be controlled in an observational study by restricting confounding matters or by adjusting the analysis. In these cases, possibly confounding conditions were noted (comorbidities) but are not really confounders because they are intermediate steps in wound development. ${ }^{[40]}$

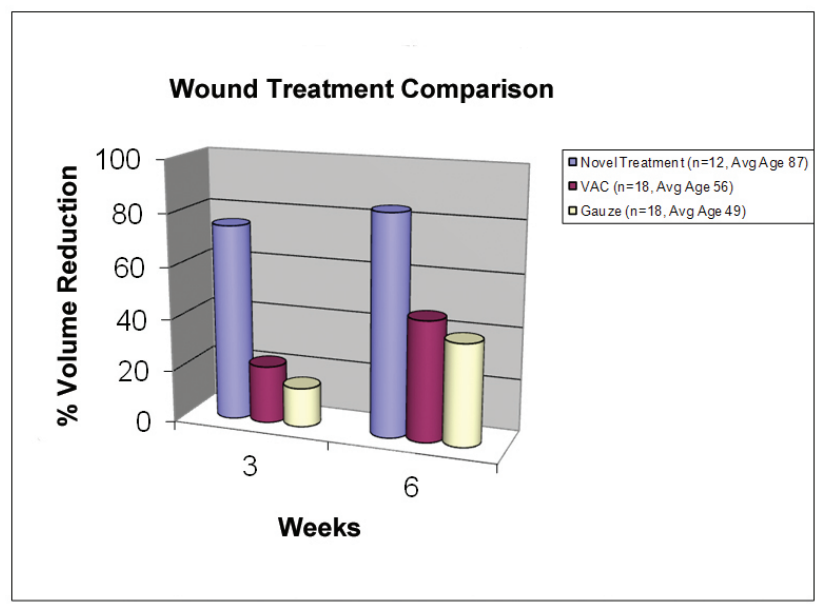

Figure 3: Comparing novel dressing treatment of hospice patients to published study of VAC $v s$. gauze for sacral/pelvic wounds. Asernip's Accelerated Review of Vacuum Assisted Wound Closure. Nov. 2005.

Statistical analysis was not done to rule out chance as a cause of results, but common sense and clinical experience would judge the results as meaningful, though not statistically significant. This study has strong internal validity and precision with lack of random error or systematic error. External validity also applies because the sample size is large and representative. However, the external vailidity will be confirmed when similar results are replicated in different populations, places, and time periods. The primary endpoint was management of wound symptoms in hospice patients. 


\section{Conclusion}

In summary, a wound treatment combination developed to care for pressure ulcers and other severe wounds, using an unexpected mixture of common ingredients resulted not only in achieving the intended goals of relieving pain, controlling odor and preventing infection, but also had the surprising and unanticipated consequence of improved wound healing. Additionally, while the treatment was developed primarily for pressure ulcers, it also proved effective for other deep wounds, such as arterial ulcers, neuropathic and venous ulcers. This ability to impact a variety of wounds is an advantage of the novel dressing that dramatically simplifies wound care. Most other dressings are developed for a particular type of wound, and the provider is required to assess and diagnose the wound in order to select the appropriate dressing. This novel dressing eliminates that need, thus making effective care much easier to provide. The results seen with this treatment method suggest that further evaluation is warranted.

\section{References}

[1] Tippett A. Wounds at the End of Life. Wounds. 2005; 17(4): 91-98.

[2] Reifsnyder J, Hoplamazian L. Incidence and Prevalence of Pressure Ulcers in Hospice. Journal of Palliative Medicine. 2005; 8(1): 244.

[3] NHPCO Facts and Figures. National Hospice and Palliative Care Organization Research Department. Alexandria. VA. Http://www . nhpco.org/files/public/Facts\%20Feb04.pdf.

[4] Cuddigan J, Berlowitz D, Ayello E. Pressure Ulcers in America: Prevalence, Incidence, and Implications for the Future: An Executive Summary of the National Pressure Ulcer Advisory Panel Monograph. Advances in Skin \& Wound Care: The Journal for Prevention and Healing. 2001 July/August; 14(4): 208-215.

[5] Berlowitz D, VanDeusen Lukas C, et al. Preventing pressure ulcers in hospitals: a toolkit for improving quality of care. Rockville, MD: Agency for Healthcare Research and Quality. 2011 http://www.ahrq.gov/professionals/systems/long-ter $\mathrm{m}$-care/resources/pressure-ulcers/pressureulcertoo lkit/index.html

[6] Allman R M. Pressure ulcer prevalence, incidence, risk factors and impact. Clinics in geriatric medicine. 1997; 13(3): 200.

[7] Garber S, Rintala D, Hart K, Fuhrer M. Pressure ulcer risk in spinal cord injury: Predictors of ulcer status over 3 years. Arch Phys Med Rehabil. 2000; 81(4): 465-471. PMid:10768537. http: $/ / \mathrm{dx}$.doi.org/10.1053/mr.2000.3889

[8] Cramp A, Warke K, Lowe-Strong A. The Incidence of Pressure Ulcers in People with Multiple Sclerosis and Persons Responsible for their Management. International Journal of MS Care. Summer 2004; 6(2): 52-54. http://dx.doi.org/10.7224/1537-2073-6.2.5 2

[9] Pressure Ulcers in Adults: Prediction and Prevention. Clinical Practice Guideline Number 3, U.S. Department of Health and Human Services, Public Health Service, Agency for Health Care Policy and Research, Rockville, MD, AHCPR Publication No. 92-0047. 1992.

[10] Treatment of Pressure Ulcers. Clinical Practice Guideline Number 15. U.S. Department of Health and Human Services, Public Health Service, Agency for Health Care Policy and Research, Rockville, MD, AHCPR Publication No. 95-0652. 1994.

[11] Reddy M, et al. Treatment of Pressure Ulcers, A Systematic Review. JAMA. 2008 December 10; 300(22): 2647-2662. PMid:19066385 http://dx.doi.org/10.1001/jama.2008.778

[12] Woo K, Abbott L, Librach L. Evidence-based approach to manage persistent wound-related pain. 2013 March; 7(1).

[13] Lindholm C. Pressure Ulcers and Infection-Understanding Clinical Features. Ostomy Wound Management. 2003 May; 49(5A): 4-7. PMid: 12883158 .

[14] Rinne C. Laying the Foundation: The Multidisciplinary Approach to Program Development. Southwest Missouri State University, FourDay Wound Management Workshop. 2001.
[15] Powell DM, et al. Damage to tissue defenses by EMLA cream. J. Emergency Medicine. 1991 Jul-Aug; 9(4): 205-9. http://dx.doi . org/10.1016/0736-4679(91) 90414-B

[16] Davies B, Guyuron B, Husami T. The role of lidocaine, epinephrine, and flap elevation in wound healing after chemical peel. Ann. Plastic Surgery. 1991; 26(3): 273-8.

[17] Drucker M, et al. Experimental Studies on the Effect of Lidocaine on Wound Healing. World J. Surg. 1998; 22: 394-398. PMid:9523522. http://dx.doi.org/10.1007/s002689900403

[18] Vasseur P B, Paul H A, Dybdal N, Crumley L. Effects of local anesthetics on healing of abdominal wounds in rabbits. Am. J. Veterinary Research. 1984; 45(11): 2385-8.

[19] Kanta J, Kopacova L, Patockova M, Bartos F. Effect of carbanilate local anesthetics on granulation tissue formation. Pol. J. Pharmacol. 1984 Nov-Dec; 36(6): 659-63. PMid:6535158.

[20] Brofeldt B T, Cornwell P, Doherty D, Batra K, Gunther R A. Topical lidocaine in the treatment of partial-thickness burns. J. Burn Care Rehabilitation. 1989 Jan-Feb; 10(1): 63-8. PMid:2921260. http://dx.doi.org/10.1097/00004630-198901000-00009

[21] Alekseev A A, et al. Activetex $(\mathbb{Q})$ Wound Dressings in Burn Treatment. Annals of Burns and Fire Disasters. 2002 March; XV(1).

[22] Christensen T, Thorum T, Kubiak E. Lidocaine Analgesia for Removal of Wound Vacuum-Assisted Closure Dressings: A Randomized Double-Blinded Placebo-Controlled Trial. Orthop Trauma 2013 February; 27(2).

[23] Rutterman M, Maier-Hasselmann A, Nink-Grebe B, Burckhardt M Local Treatment of Chronic Wounds. Deutsches Arzteblatt International. 2013; 110(3): 25-31.

[24] Brolmann F, et al. Evidence-based decisions for local and systemic wound care. British Journal of Surgery. 2012; 99: 1172-1183. PMid:22777856. http://dx.doi.org/10.1002/bjs.8810

[25] Hollmann M W, Durieux M E, Fisher D M. Review Article: Local Anesthetics and the Inflammatory Response. Anesthesiology. 2000 Sept.; 93(3). http://dx.doi.org/10.1097/00000542-2 00009000-00038

[26] Kaye E T. Antibacterial Therapy: Topical Antibacterial Agents Infectious Disease Clinics of North America. 2000 June; 14(2). http://dx.doi.org/10.1016/S0891-5520(05)70250-6

[27] Laboratory testing of inhibition of novel dressing against a panel of micro-organisms, using Kirby-Bauer Standard Antimicrobial Susceptibility Test, conducted by NAMSA. April 2006.

[28] Purcell A, Marshall A, King J, Buckley T. Eutectic mixture of local anaesthetics (EMLA) 5\% cream as a primary dressing on a painful lower leg ulcer. Journal of Wound Care. 2012 July; 21(7): 309. PMid:22886329. http://dx.doi.org/10.12968/jowc. 2 012.21 .7 .309

[29] Berg J, et al. Antibacterial properties of EMLA and lidocaine in wound tissue biopsies for culturing. Wound Rep Reg. 2006; 14: 581585. PMid:17014670. http://dx.doi .org/10.1111/j.1743-6 109.2006.00157.x

[30] Poteete V. Case study: eliminating odors from wounds. Decubitus. 1993 Jul; 6(4): 43-6. PMid:8297491. 
[31] Wintergreen Essential Oil is Mother Nature's Aspirin. http://www .experience-essential-oils.com/winter green-essential-oil.html.Accessed11/10/2013.

[32] Methyl salicylate compound summary. National Center for Biotechnology Information. http://pubchem.ncbi.nlm.nih.gov/sum mary/summary.cgi?cid=4133referenceforoilof wintergr een

[33] Patel B, Cox-Hayley D. Managing Wound Odor. Fast Facts and Concepts. August 2009; 218. Available from: http://www . eperc.mcw . edu/EPERC/FastFactsIndes/f ff_sa8.htm.Accessed11/10/13.

[34] Kalinski, et al. Effect of Metronidazole $0.75 \%$ Gel on Wound Odor: Summary of Investigator and Patients' Scores. WOUNDS. 2005; 17(4): 84-90.

[35] Bolton L. Evidence Corner: Radiant Heat Therapy and Chronic Wound Healing. Wounds. 2005; 17(10): A8-A8.
[36] Asernip S. Accelerated review of Vacuum Assisted Wound Closure. Appendix A: Key Efficacy and Safety Findings-Randomized Controlled Trials. 2003.

[37] Milne S, Connolly P. The influence of different dressings on the $\mathrm{pH}$ of the wound environment. Journal of Wound Care. 2014 February; 23(2): 53-57. PMid:24526080. http://dx.doi.org/10.12968/ jowc. 2014.23.2.53

[38] Schim S, Cullen B. Wound Care at End of Life. Nursing Clinics of North America. 40(2): 281-294. PMid:15924895.

[39] Carlson M, Morrison R. Study Design, Precision, and Validity in Observational Studies J. Palliative Medicine. Jan 2009; 12(1): 77 82. PMid:19284267. http://dx.doi.org/10.1089/jpm. 2008. 9690

[40] Jepsen P, Johnsen S, Gillman M, Sorensen H. Interpretation of observational studies. Heart. Aug 2004; 90(8): 956-960. PMid:15253985. http://dx.doi.org/10.1136/hrt.2003.01 7269 